\title{
Clinicopathological study of 35 cases of multiple system atrophy
}

\author{
G K Wenning, Y Ben-Shlomo, M Magalhães, S E Daniel, N P Quinn
}

\begin{abstract}
The clinical and pathological features of 35 cases with multiple system atrophy collected in the United Kingdom Parkinson's Disease Society Brain Bank (UKPDSBB) between 1985 and 1992 have been analysed. The median age of onset was 55 (range $33 \cdot 3-75 \cdot 8$ ) years and median survival was $7 \cdot 3$ (range $2 \cdot 1-11 \cdot 5$ ) years. Parkinsonism, usually asymmetric, occurred in all, and autonomic failure in all but one case. Cerebellar signs were noted in $34 \%$ and pyramidal features in $54 \%$ of the cases.

Glial cytoplasmic inclusions were found in all cases with adequate fixation. Lewy bodies were detected in three cases. The substantia nigra was (usually severely) depleted of cells in all cases. With two exceptions the putamen was atrophic; the caudate and pallidum were less commonly and less severely affected.

Overall nigrostriatal cell loss correlated with severity of disease at the time of death. The latest, but not the best, recorded levodopa response tended to be inversely related to the degree of putaminal degeneration. The olivopontocerebellar system was involved in $88 \%$ of the cases, the cerebellar vermis usually being more severely affected than the hemispheres. The presence of associated cerebellar pathology was, however, unrelated to the presence of cerebellar signs in life.
\end{abstract}

(F Neurol Neurosurg Psychiatry 1995;58:160-166)

Keywords: multiple system atrophy; striatonigral degeneration; olivopontocerebellar atrophy.

Multiple system atrophy is a sporadic degenerative disease of the nervous system that causes any combination of parkinsonism, autonomic and cerebellar dysfunction, and pyramidal signs. ${ }^{1-3}$ Pathologically, it is characterised by varying degrees of cell loss and gliosis principally affecting the substantia nigra and putamen (striatonigral degeneration), pontine nuclei, cerebellar Purkinje cells, and inferior olives (olivopontocerebellar atrophy), intermediolateral cell columns of the thoracic spinal cord, and Onuf's nucleus in the sacral cord. ${ }^{45}$ The medical literature contains more than 300 pathologically established cases. Most reports involve only a few cases, however, or give limited clinical information to correlate with the pathological findings.

\section{Materials and methods}

The United Kingdom Parkinson's Disease Society Brain Bank (UKPDSBB) had received 370 brains of patients with parkinsonism by October 1992 . Of these, 35 (9.5\%) showed the pathological changes of multiple system atrophy and form the basis of this paper. Thirteen of these patients, all with a clinical diagnosis of multiple system atrophy, had been seen in life by one of us (NPQ). The remaining 22 had been followed up by other physicians, 17 of whom were neurologists. Clinicopathological details of nine and an assessment of levodopa response in 21 of these cases have previously been reported. ${ }^{67}$

\section{CLINICAL DATA}

The clinical features of the 35 cases were abstracted from clinical records by GKW and entered on a database for subsequent analysis. If no mention of a clinical feature could be found in the notes, then the feature was coded as absent. In some cases, a patient may have had such a feature but this was either not evaluated or recorded. Hence the proportions of patients with the presence of a feature should be treated as a "conservative" estimate.

Autonomic failure was defined as the presence of any of the following: symptomatic postural hypotension, urinary urge incontinence, faecal incontinence, urinary retention requiring catheterisation, or persistent erectile failure. Severe autonomic failure, for men, was defined as two or more of the following five symptoms: syncope, urinary incontinence, faecal incontinence, urinary retention, and erectile failure. For women, the definition of severe autonomic failure was based on two or more of the symptoms, excluding erectile failure.

Akinesia (usually with rigidity) was necessary for a diagnosis of parkinsonism. Tremor was neither obligatory, however, nor sufficient on its own. The Hoehn and Yahr stage for cases was derived from the notes. Early loss of balance was defined as Hoehn and Yahr stage III or worse within three years of onset of symptoms. Response to levodopa, based on the recorded estimate in the records of either the patient, or physician, or both, was graded on a four point scale $(1=0-29 \%, 2=$ $30-49 \%$ (both poor), $3=50-69 \%$ (good), 4 $=70-100 \%$ (excellent)). Initial, best, and latest recorded response to levodopa were noted. Response data on four cases were missing.

Cerebellar features that were recorded included limb ataxia, intention tremor, gait ataxia, and nystagmus, in isolation or in 
Figure 1 Frequency of various combinations of clinical features in 35 cases of pathologically confirmed multiple system atrophy.

Park = parkinsonism; Auto = autonomic failure; Cereb = cerebellar signs; Pyr = pyramidal signs.

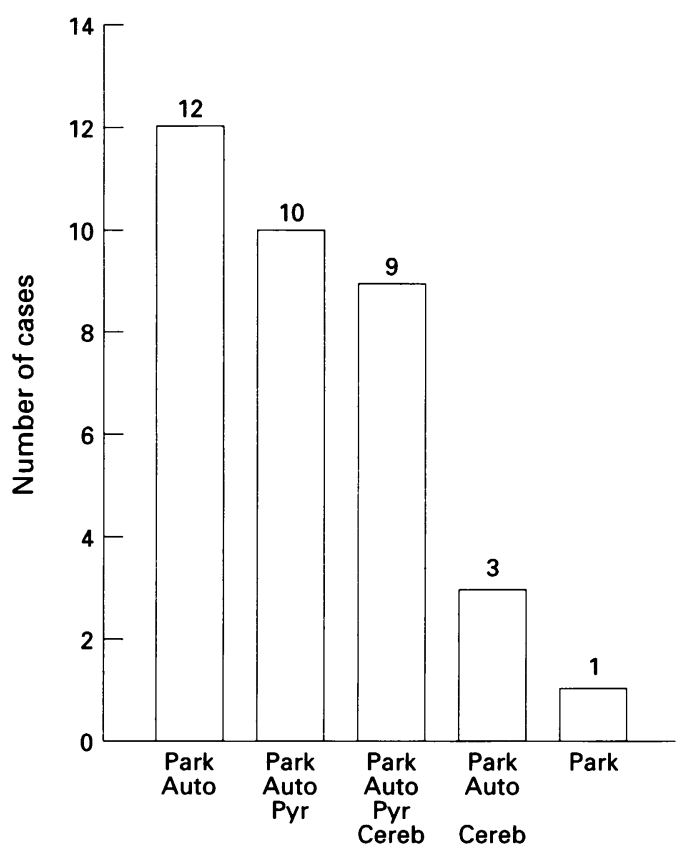

combination. Pyramidal features comprised equivocal or extensor plantar responses and hyperreflexia. Equivocal plantar responses and hyperreflexia had to occur together to be accepted as pyramidal signs.

Patients were diagnosed as clinically probable multiple system atrophy of striatonigral degeneration type if parkinsonism was the only (pure striatonigral degeneration type) or predominant (predominant striatonigral degeneration type) motor syndrome. Multiple system atrophy of olivopontocerebellar type was diagnosed if a cerebellar syndrome was the only (pure olivopontocerebellar atrophy type) or predominant (predominant olivopontocerebellar atrophy type) motor syndrome.

In addition, clinical notes were screened for a variety of other symptoms or signs including diplopia, hypometric saccades, reduced horizontal or vertical gaze, dysarthrophonia, dysphagia, excessive snoring, respiratory stridor, myoclonus, disproportionate antecollis, and sensory dysfunction (subjective or objective). Age at onset of autonomic failure, parkinsonism, cerebellar, and pyramidal signs were recorded for analysis of the evolution of multiple system involvement in multiple system atrophy.

\section{POSTMORTEM MATERIAL}

All cases were subject to full neuropathological examination by routine techniques. Tissue blocks were taken from the cerebrum, brainstem, and cerebellum, and a variety of histological stains were used, including haematoxylin-eosin, luxol fast blue, modified Bielschowsky silver impregnation, and immunocytochemistry for glial fibrillary acidic protein and ubiquitin.

A pathological diagnosis of multiple system atrophy was based on cell loss and gliosis in any two of the following structures: striatum, substantia nigra, locus ceruleus, other pontine nuclei, cerebellar Purkinje cells, and inferior olives, together with the presence of oligoden- droglial cytoplasmic inclusions on modified Bielschowsky silver impregnation or antiubiquitin staining. ${ }^{89}$ As it was available for only nine of the 35 cases, we have not included spinal cord pathology in this paper.

The average degree of cell loss and gliosis in the affected parts (severity) was semiquantitatively rated by one of the authors (SED), without knowledge of clinical features, on a 4 point scale ranging from normal $(0)$ to severe (3) in the following sites: putamen, caudate nucleus, globus pallidus, substantia nigra, locus ceruleus, pontine nuclei, inferior olives, and cerebellar Purkinje cells. In addition to the degree of cell loss (severity), the area of putaminal damage was assessed by dividing the lateromedial extent of the putamen at the level of the anterior commissure into eight segments and expressing the area of damage as the number of segments ( 0 to 8 ) involved. A total measure for putaminal damage was obtained by multiplying the severity of damage ( 0 to 3 ) by area ( 0 to 8 ) to give a total putaminal score (range 0 to 24). Nigral and putaminal severity counts were combined as a striatonigral degeneration score (range 0 to 6 ), and severity counts in inferior olives, pontine nuclei, and cerebellar Purkinje cells as an olivopontocerebellar atrophy score (range 0 to 9 ).

\section{STATISTICAL ANALYSIS}

In presenting data for continuous variables, we have used median values as these were not always normally distributed. Non-parametric tests of significance have been carried out with the Wilcoxon-Mann-Whitney test for continuous or nominal variables, but for ease of interpretation mean values have been shown when comparing nominal data on pathological severity. Correlations were calculated with the Spearman correlation coefficient, and exact confidence intervals (95\%) were calculated for proportions with the binomial formula. ${ }^{10}$ Contingency tables were analysed with either a $\chi^{2}$ test or Fisher's exact test when the smallest expected value was less than five. All significance tests are two sided and use an $a$ value of 0.05 .

\section{Results}

\section{CLINICAL FEATURES}

There were 19 men and 16 women (ratio $1 \cdot 2: 1)$. Median age at disease onset was 55 (range $33 \cdot 3-75 \cdot 8$ ) years. Death was at $62 \cdot 8$ (range $39 \cdot 3-81 \cdot 3$ ) years, and median survival was $7 \cdot 3$ (range $2 \cdot 1-15$ ) years. There were no significant differences in any of these variables by sex. Thirty $(86 \%)$ of the patients were considered to have multiple system atrophy of the striatonigral degeneration type, and five (14\%) multiple system atrophy of the olivopontocerebellar atrophy type (table 1 ).

The proportion of cases with some evidence of abnormalities in four major systems were as follows: autonomic 97 (95\% confidence interval (95\% CI) $85-100) \%$, parkinsonian 100 (95\% CI 90-100)\%, cerebellar 34 (95\% CI 19-52)\%, and pyramidal 54 (95\% CI 
Table 1 Distribution of subgroups among 35 cases of multiple system atrophy

\begin{tabular}{|c|c|c|c|c|c|}
\hline SND & 30 & Pure SND & 23 & \multirow{4}{*}{ Mixed } & \\
\hline & & Predominant SND & 7 & & \\
\hline \multirow{2}{*}{ OPCA } & \multirow{2}{*}{5} & Predominant OPCA & 5 & & \\
\hline & & Pure OPCA & 0 & & \\
\hline
\end{tabular}

SND $=$ Striatonigral degeneration; OPCA $=$ olivopontocerebellar degeneration.

Table 2 Basic clinical features in 35 pathologically established cases of multiple system atrophy

\begin{tabular}{lrl} 
& \multicolumn{2}{c}{ Cases with } \\
& \multicolumn{2}{c}{ feature recorded } \\
\cline { 2 - 3 } & $\%$ & \multicolumn{1}{c}{$(95 \% \mathrm{CI})$} \\
\hline Parkinsonism & 100 & $(90-100)$ \\
Akinesia/rigidity & 100 & $(90-100)$ \\
Tremor & 80 & $(63-92)$ \\
Present at rest & 34 & $(19-52)$ \\
Classical & 11 & $(3-26)$ \\
Postural & 29 & $(15-46)$ \\
Action & 9 & $(2-23)$ \\
Cerebellar features & 34 & $(19-52)$ \\
Limb ataxia & 31 & $(17-49)$ \\
Intention tremor & 11 & $(3-27)$ \\
Gait ataxia & 29 & $(15-46)$ \\
Postural instability & 89 & $(73-97)$ \\
Nystagmus & 14 & $(5-30)$ \\
Autonomic features & 97 & $(85-100)$ \\
Recurrent syncope ( $\geqslant 3$ episodes) & 20 & $(8-37)$ \\
Symptomatic postural hypotension & 51 & $(34-69)$ \\
Urinary incontinence & 51 & $(34-69)$ \\
Urinary retention & 34 & $(19-52)$ \\
Faecal incontinence & 3 & $(0-19)$ \\
Impotence (men) - see also text & 62 & $(32-86)$ \\
Pyramidal features & 54 & $(37-71)$ \\
Extensor plantars & 37 & $(22-55)$ \\
Equivocal plantars & 17 & $(7-34)$ \\
Hyperreflexia & 57 & $(39-74)$ \\
\hline
\end{tabular}

$36 \cdot 7-71 \cdot 2) \%$. Figure 1 shows the pattern of involvement of these four systems. The most frequent combination of clinical features was autonomic involvement with parkinsonism. Of the 35 cases, $22(63 \%)$ had involvement of three or more systems; the remainder only showed clinical features of parkinsonism with $(n=12)$ or without $(n=1)$ autonomic involvement.

Table 2 provides more detail on the presence of specific clinical features. Akinesia and rigidity were present in all patients and were usually asymmetric throughout the disease course. Tremor at rest was seen in a third of patients; however, a classical pill rolling rest tremor had been documented in only $11 \%$.

Table 3 Other clinical features

\begin{tabular}{lrl}
\hline & \multicolumn{2}{c}{ Cases with feature recorded } \\
\cline { 2 - 3 }$\%$ & \multicolumn{1}{c}{$(95 \%$ CI } \\
\hline Diplopia & 14 & $(5-30)$ \\
Hypometric saccades & 29 & $(15-46)$ \\
Reduced upgaze & 20 & $(8-37)$ \\
Reduced downgaze & 9 & $(2-23)$ \\
Reduced horizontal gaze & 9 & $(2-23)$ \\
Dysarthophonia & 89 & $(73-97)$ \\
Dysphagia & 51 & $(34-69)$ \\
Excessive snoring & 17 & $(7-34)$ \\
Stridor & 34 & $(19-52)$ \\
Myoclonus & 29 & $(15-46)$ \\
Disproportionate antecollis & 9 & $(2-23)$ \\
Sensory dysfunction & 20 & $(8-37)$ \\
$\quad$ Symptoms only & 11 & $(3-27)$ \\
Signs \pm symptoms & 9 & $(2-23)$ \\
\hline
\end{tabular}

All but one patient had autonomic failure according to our definition. Although symptomatic postural hypotension was noted in $71 \%$ of the patients this was rarely severe, with recurrent $(\geqslant 3)$ syncopal attacks occurring in only $20 \%$. Urinary urge incontinence occurred in half of the patients, but faecal incontinence in one patient only. Urinary retention was less common, affecting a third of the patients. Persistent erectile dysfunction was reported by almost two thirds of men; there was no mention of potency in the remaining third.

Cerebellar gait ataxia and limb ataxia were seen in about $30 \%$ of the patients, and pyramidal signs were detected in $54 \%$.

Table 3 shows the prevalence of additional features. Reduced downgaze was noted in three $(9 \%)$ patients; however, this deficit was only mild, and none of the cases had a prominent supranuclear downgaze palsy. Dysarthrophonia affected all but four patients and was commonly atypical with elements of straining, quivering, slurring, or scanning as well as the usual hypophonia and monotony of parkinsonism. Recorded sensory symptoms were uncommon (seven cases) and sensory loss even rarer (three cases).

When comparing sex distribution, age of onset, age at death, duration of disease, and response to levodopa for clinical subgroups, cases with cerebellar features $(34 \%)$ were more likely to be men $(p=0.001)$, have a younger age of onset $(p=0.0005)$, and younger age of death $(p=0.003)$, but survival was little different from the other subgroups. Cases with severe autonomic involvement $(37 \%)$ were also more likely to have a younger age of onset $(p=0.02)$ and of death $(p=$ 0.05 ) and tended to have a poor response to levodopa although this failed to reach statistical significance $(p=0.06)$

Levodopa was given to all but two patients. The initial clinical response for the whole sample was excellent (4) in $13 \%$, good (3) in $25 \%$, and poor (1-2) in $62 \%$. The best recorded levodopa response was excellent (4) in $13 \%$, good (3) in $32 \%$, and poor (1-2) in $55 \%$. The last recorded response in the notes, however, showed only $7 \%$ with a good response and $93 \%$ with a poor response. Motor response fluctuations were recorded in $60 \%$ of treated patients. Dyskinesiae developed in $52 \%$ of patients, involving facial musculature in $59 \%$ ( $12 \%$ unilateral) and limbs in $53 \%$ of them. Psychiatric side effects occurred in $15 \%$ (confusion in $9 \%$, hallucinations and nightmares in $6 \%$ each).

Figure 2 shows the disease progressionnamely, the median number of years to reach different Hoehn and Yahr stages in these cases with multiple system atrophy in comparison with published data from 123 treated cases of Parkinson's disease. ${ }^{11}$ It is clear that the progression of disability in multiple system atrophy is faster than that seen in Parkinson's disease, the difference being most pronounced for latency to reach stage III (bilateral disease plus postural instability).

Initially 26 cases $(74 \%)$ were thought to 
Figure 2 Median number of years to reach different Hoehn and Yahr stages for 35 cases of pathologically' confirmed multiple system atrophy (empty bars) compared with 123 patients with clinically diagnosed Parkinson's disease (black bars). Data for Parkinson's disease taken from Hoehn. "Bars indicate range.

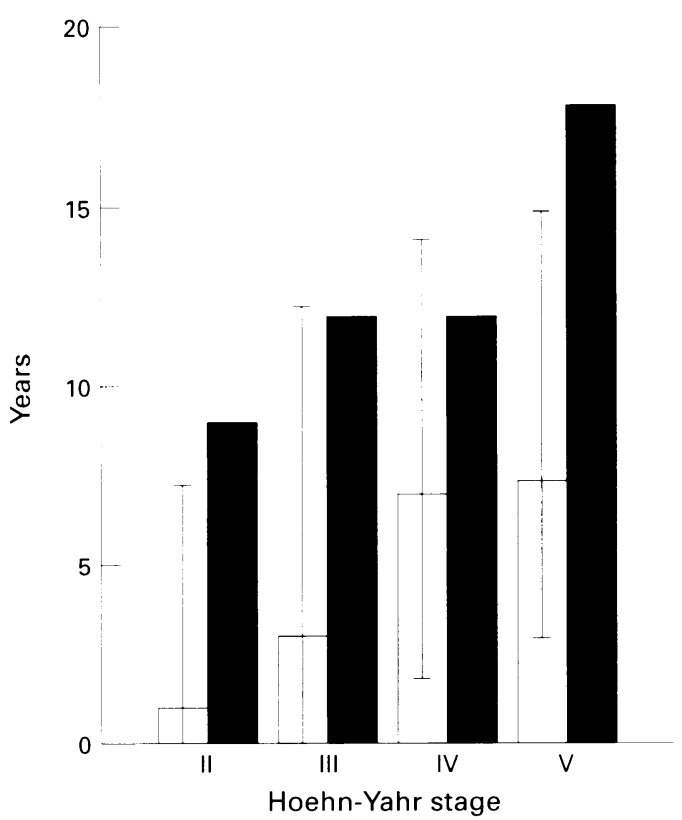

have idiopathic Parkinson's disease. Among them, $14(40 \%)$ subsequently had their diagnosis changed to multiple system atrophy after a median disease duration of $4 \cdot 4$ $(0 \cdot 3-7 \cdot 8)$ years. None of our own 13 cases, but $12(55 \%)$ of the other 22 cases, died still carrying an erroneous diagnosis of Parkinson's disease. We examined whether, even in retrospect, any of these 12 cases had any atypical features recorded that might suggest the diagnosis of multiple system atrophy. In none of them had cerebellar features or evi-

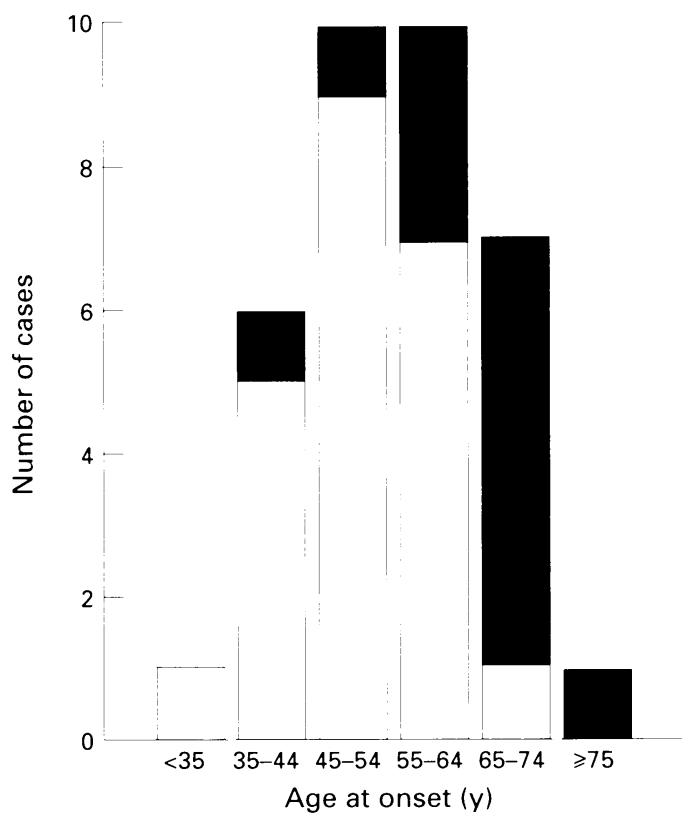

Figure 3 Age of onset distribution for 35 cases of multiple system atrophy according to final clinical diagnosis (multiple system atrophy' or Parkinson's disease). Empty' bars indicate patients diagnosed as multiple system atrophy in life, black bars indicate patients misdiagnosed as

Parkinson's disease.

dence of severe autonomic involvement been noted in life, but two had pyramidal features. Their age of onset was significantly older than the 23 correctly diagnosed cases (63.4 $v 50.6$ years $p=0.002$, fig 3 ), and there was a nonsignificant trend for better levodopa response in this group.

Table 4 Pathological findings in 35 cases of multiple system atrophy

\begin{tabular}{|c|c|c|c|c|c|c|c|c|c|c|c|c|c|c|}
\hline Case & $\begin{array}{l}S N D \\
\text { score } \\
(0-6)\end{array}$ & $\begin{array}{l}\text { OPCA } \\
\text { score } \\
(0-9)\end{array}$ & $C C$ & $C N$ & $P u$ & $\begin{array}{l}\text { Pu score } \\
(0-24)\end{array}$ & $\mathrm{Pa}$ & $S_{n}$ & $L B$ & $L i$ & $P N$ & $I O$ & $C P$ & \\
\hline 1 & 2 & 0 & - & + & - & 0 & + & +++ & - & $t+t$ & - & - & + & \\
\hline 2 & 3 & 1 & - & - & - & 0 & - & +++ & - & $++t$ & - & - & + & \\
\hline 3 & 4 & NA & - & - & + & 1 & - & +++ & - & +++ & ++ & $++t$ & $\mathrm{NE}$ & \\
\hline 4 & 4 & NA & - & - & + & 1 & - & +++ & - & + & + & NA & + & $\mathrm{V}>\mathrm{H}$ \\
\hline 5 & 4 & 4 & - & + & ++ & 4 & ++ & ++ & (5) & +++ & + & + & ++ & $\mathrm{V}>\mathrm{H}$ \\
\hline 6 & 4 & 9 & NA & - & ++ & 6 & +++ & ++ & - & +++ & +++ & +++ & +++ & $\mathrm{V}>\mathrm{H}$ \\
\hline 7 & 4 & 9 & - & + & ++ & 4 & - & ++ & - & ++ & +++ & +++ & $+\cdots+$ & $\mathrm{V}=\mathrm{H}$ \\
\hline 8 & 5 & 4 & - & ++ & ++ & 8 & + & $+\cdots+$ & - & - & ++ & - & $+\cdot$ & $\mathrm{V}>\mathrm{H}$ \\
\hline 9 & 5 & 5 & - & + & ++ & 6 & + & $++t$ & $(6)$ & ++ & - & ++ & $+\cdots+$ & $\mathrm{V}>\mathrm{H}$ \\
\hline 10 & 5 & 6 & NA & + & ++ & 8 & + & +++ & - & +++ & + & +++ & ++ & \\
\hline 11 & 5 & 7 & - & + & +++ & NA & + & ++ & - & $+t$ & ++ & ++ & +++ & \\
\hline 12 & 5 & 8 & NA & - & +++ & NA & - & +++ & - & - & +++ & +++ & $+\cdots$ & \\
\hline 13 & 5 & 9 & - & - & +++ & NA & ++ & ++ & - & +++ & +++ & +++ & $+\cdots+$ & \\
\hline 14 & 5 & 9 & - & - & ++ & 6 & + & $+\cdots+$ & - & +++ & +++ & +++ & +++ & \\
\hline 15 & 6 & NA & - & - & +++ & $\mathrm{NA}$ & ++ & $+\cdots+$ & - & +++ & $\mathrm{NE}$ & NA & + & \\
\hline 16 & 6 & NA & - & ++ & +++ & 21 & ++ & $++t$ & - & -++ & +++ & ++ & $\mathrm{NA}$ & \\
\hline 17 & 6 & 0 & - & ++ & +++ & 18 & ++ & +++ & - & +++ & -- & - & (7) & \\
\hline 18 & 6 & 0 & - & ++ & +++ & 18 & - & +++ & - & $+t+$ & - & + & (7) & \\
\hline 19 & 6 & 0 & - & - & +++ & $\mathrm{NA}$ & NE & $++t$ & -- & ++ & - & - & - & \\
\hline 20 & 6 & 0 & - & t & +++ & 24 & ++ & +++ & - & +++ & - & - & - & \\
\hline 21 & 6 & 2 & (1) & ++ & $++\cdots$ & 9 & ++ & +++ & +- & -++ & - & - & + & $\mathrm{V}=\mathrm{H}$ \\
\hline 22 & 6 & 2 & NÁ & ++ & +++ & 24 & ++ & +++ & - & ++ & - & - & + & $\mathrm{V}=\mathrm{H}$ \\
\hline 23 & 6 & 2 & (2) & + & +++ & 18 & + & +++ & $+t$ & ++ & - & + & + & $\mathrm{V}=\mathrm{H}$ \\
\hline 24 & 6 & 3 & - & +++ & +++ & 24 & +++ & $++t$ & - & $t+t$ & t & + & t & \\
\hline 25 & 6 & 4 & - & ++ & +++ & 21 & + & $+t+$ & - & +++ & + & + & ++ & $\mathrm{V}>\mathrm{H}$ \\
\hline 26 & 6 & 5 & - & + & +++ & 23 & + & $++t$ & - & +++ & - & ++ & $+\cdots+$ & $\mathrm{V}>\mathrm{H}$ \\
\hline 27 & 6 & 5 & - & ++ & +++ & 21 & + & $+\cdots+$ & - & -++ & - & + & $+\cdots+$ & $\mathrm{V}>\mathrm{H}$ \\
\hline 28 & 6 & 5 & - & +++ & +++ & 24 & ++ & +++ & - & -++ & + & ++ & +- & $\mathrm{V}>\mathrm{H}$ \\
\hline 29 & 6 & 6 & - & + & +++ & 18 & + & +++ & - & -++ & ++ & - & +++ & $\mathrm{V}>\mathrm{H}$ \\
\hline 30 & 6 & 6 & (3) & + & +++ & 21 & + & $++t$ & + & $++t$ & + & +++ & +++ & $\mathrm{V}>\mathrm{H}$ \\
\hline 31 & 6 & 9 & (4) & ++ & +++ & 24 & ++ & +++ & - & $++t$ & +++ & +++ & +++ & $\mathrm{V}=\mathrm{H}$ \\
\hline 32 & 2 & NA & NA & + & + & 1 & - & t & - & ++ & - & - & $\mathrm{NE}$ & \\
\hline 33 & NA & NA & - & ART & ++ & NA & ART & ART & - & ART & ART & ART & ART & \\
\hline 34 & $\mathrm{NA}$ & $\mathrm{NA}$ & NA & INF & INF & INF & $\mathrm{NE}$ & + & - & $\mathrm{NA}$ & - & ART & ART & \\
\hline 35 & $\mathrm{NA}$ & 4 & - & INF & INF & INF & +++ & +++ & - & +++ & - & + & $+\cdots+$ & $\mathrm{V}>\mathrm{H}$ \\
\hline
\end{tabular}

SND = Striatonigral degeneration; OPCA = olivopontocerebellar atrophy (for scores refer to methods); CC = cerebral cortex; $\mathrm{CN}=$ caudate nucleus; $\mathrm{Pu}=$ putamen; $\mathrm{Pa}=$ pallidum; $\mathrm{Sn}=$ substantia nigra; $\mathrm{LB}=$ Lewy bodies; $\mathrm{PN}=$ pontine nuclei; $\mathrm{TF}$ transverse fibres; $\mathrm{IO}=$ inferior olives; $\mathrm{CP}=$ cerebellar Purkinje cells; $\mathrm{V}=$ vermis; $\mathrm{H}=$ hemisphere;,,,$-++++++=$ absent, mild, moderate, severe cell loss \pm gliosis; NA $=$ not available; NE = not examined; ART $=$ artefact; INF $=$ infarction;
$(1),(2),(3)=$ LBs present in $\mathrm{CC} ;(4)=$ Alzheimer type change in CC; (5) = pale inclusion bodies in $\mathrm{Sn} ;(6)=$ eosinophilic degeneration present; (7) = agonal change 
PATHOLOGY

Cell loss in the substantia nigra was identified in all but one case, which showed postmortem artefact (table 4), and was severe in $79 \%$ $(27 / 34)$ of them. The locus ceruleus was, with few exceptions, equally severely depleted.

Putaminal cell loss and gliosis were pronounced in $20(61 \%)$ and moderate or mild in 11 cases $(33 \%)$. In two cases there was no putaminal cell loss and in two others infarction made interpretation of the extent of degenerative change difficult. Nevertheless, in all four cases both substantia nigra and locus ceruleus showed cell loss and gliosis without Lewy bodies. The pathological diagnosis of multiple system atrophy in the cases without putaminal cell loss would not have been possible but for the demonstration of glial cytoplasmic intrusions. ${ }^{12}$

The caudate nucleus was much less severely affected: only two (6\%) cases had severe cell loss and gliosis (usually diffuse) and $21(66 \%)$ cases had mild or moderate gliosis, often in a focal dorsolateral distribution. There were no changes in nine $(28 \%)$ cases. The external pallidum was invariably involved with gliosis except for the two cases without putaminal cell loss.

Degenerative changes in the olivopontocerebellar system were seen in 29 of $34(88 \%)$ cases studied. Assessment was not possible in one case due to postmortem artefact. Pronounced Purkinje cell depletion was seen in the cerebellar cortex of $12(40 \%)$ of the cases, and only two (6\%) cases appeared to have no cell loss at all. When present, cell loss was more severe in the cerebellar vermis relative to hemispheres in 12 of $17(71 \%)$ cases in which comparisons were possible. The dentate nuclei were only mildly affected by gliosis, without cell loss, in seven of 20 (35\%) cases in which we were able to examine them. Dentate hilum and superior cerebellar peduncle were unaffected. The dorsal vagal nucleus was mildly to severely affected in 14 of 27 $(52 \%)$ cases examined.

Finally, apart from the presence of glial cytoplasmic inclusions and age related neuritic plaques, the cerebral cortex was normal in 25 out of $30(83 \%)$ cases studied. Three $(10 \%)$ cases showed widespread Lewy bodies in all areas of cerebral neocortex studied as well as in the substantia nigra. Severe Alzheimer's disease type changes were seen in the neocortex in two $(7 \%)$ cases.

CLINICOPATHOLOGICAL CORRELATION

There was no correlation between semiquantitative measures of pathological damage in the putamen, nigra, and olivopontine area and age at onset, age at death, and duration of disease. There was a trend, however, for the degree of nigral and putaminal damage to be greater in those cases with longer disease duration.

Only $18 \%(3 / 17)$ patients with a putaminal damage score of 18 or less died with a recorded Hoehn and Yahr stage of $\mathrm{V}$ by contrast with $70 \%(7 / 10)$ of those with putaminal damage of more than $18(\mathrm{p}<0.02)$. The combined score of striatonigral degeneration and olivopontocerebellar atrophy did not, however, correlate with severity of motor disability.

Thirty five per cent of patients with a putaminal score $\leqslant 18$ showed either a good $(12 \%$, $2 / 17)$ or moderate $(24 \%, 4 / 17)$ latest recorded levodopa response whereas only one of 10 (10\%) patients with a putaminal damage score of $\geqslant 18$ retained a moderate response, but this was not significant $(p=0 \cdot 20)$. There was no relation between the latest levodopa response and degree of nigral degeneration.

The estimated degree of olivopontocerebellar atrophy was unrelated to levodopa responsiveness. The presence of fluctuations and dyskinesiae was more often associated with severe striatonigral (striatonigral degeneration score 6 ) or putaminal (putamen score $>18$ ) degeneration.

Cerebellar signs had been noted in life in only nine of the 26 (35\%) cases that showed cerebellar Purkinje cell loss at postmortem. In 12 cases this Purkinje cell loss predominated in the vermis. Although all of them were noted to have postural instability in life, only three of them had definite evidence of gait ataxia.

We also examined the association between pathological damage and clinical features and subgroups. All the cases had parkinsonism and all showed pronounced damage to the nigra, and cases with cerebellar features or classified as olivopontocerebellar atrophy type had greater damage in the olivopontine area. Perhaps surprisingly, early loss of balance correlated significantly with putaminal damage but not with the olivopontocerebellar atrophy pathological score.

\section{Discussion}

The nosology of multiple system atrophy is complex, and related to the relative predominance of parkinsonism (striatonigral degeneration), ${ }^{13}$ cerebellar signs (olivopontocerebellar atrophy), ${ }^{14}$ or autonomic failure. Shy and Drager $^{14}$ reported two patients; pathology, which showed the classic changes of multiple system atrophy (both striatonigral degeneration and olivopontocerebellar atrophy), was obtained in one of them. Over subsequent years, the term Shy-Drager syndrome ${ }^{15}$ has been used for cases of multiple system atrophy, and also misused to describe cases of Lewy body parkinsonism associated with severe autonomic failure. As virtually all patients with multiple system atrophy have some degree of clinical autonomic dysfunction (usually mild cardiovascular and more severe bladder involvement), we believe that it is most useful to divide them, according to their motor disorder, into parkinsonism predominant (striatonigral degeneration) or cerebellar predominant (olivopontocerebellar atrophy).

This study confirms and extends our knowledge about multiple system atrophy in a number of areas. It is a rapidly progressive disease, with a median survival of only five to 
six years from first symptom in over 200 cases of pathologically established multiple system atrophy recorded in the literature. ${ }^{8}$ In this series we found a longer median survival, of $7 \cdot 3$ (range $2 \cdot 1$ to 15 ) years, a discrepancy that might be due to the relative lack of clinical data in many of the published case reports. Moreover, in a follow up study of 100 patients with clinically probable multiple system atrophy we found a still longer median survival of 9.5 years. ${ }^{16}$ The total disease duration from first symptom to death for our five patients with predominant olivopontocerebellar atrophy was similar to that for the 30 patients with predominant striatonigral degeneration, and the response of their (by definition mild or moderate) parkinsonism to levodopa was uniformly disappointing.

The finding of parkinsonism in $100 \%$ of our cases comes as little surprise given that the source of our material was a parkinsonian brain bank, but parkinsonism was also reported in $89 \%$ of pathologically established multiple system atrophy cases in the literature. Almost all patients with multiple system atrophy in our series had developed clinical signs of multisystem involvement by the time of death. Apart from one $(2.9 \%)$ case with isolated parkinsonism, there was no case with autonomic failure, cerebellar, or pyramidal signs persisting in isolation throughout the course of disease. Among pathologically established cases of multiple system atrophy in the literature $90 \%$ had accrued in life features in two or more of these four clinical domains and only $10 \%$ apparently had parkinsonism in isolation at the time of death.

A good or excellent initial motor response to levodopa was seen in a third of this series of patients with multiple system atrophy. This was sustained until death, however, in only $7 \%$ of them. Thus although encountered in only a few patients, a positive and sustained levodopa response, even until death in some cases, can occur in multiple system atrophy. In this series there was a trend for the last, but not best, recorded levodopa response to be inversely associated with the degree of putaminal damage, consistent with previous more detailed morphometric data in a subgroup of cases. ${ }^{6}$ This finding might suggest that the severity of the nigral lesion precedes that of the striatal one. Further evidence for initial and more severe nigral cell loss is derived from two cases in our series with "minimal change" multiple system atrophy in which severe neuronal cell loss in substantia nigra was associated with preserved putaminal neurons ${ }^{12}$; however, typical glial cytoplasmic inclusions were found throughout the striatum and many other sites, indicating extranigral subcellular pathology. One of these two patients had a good, and one a negligible, response to levodopa. The substrate of levodopa unresponsiveness in multiple system atrophy is uncertain. Although loss of striatal dopamine receptors plays a part, much of the levodopa resistance in multiple system atrophy, and its heterogeneity between patients, is probably due to the variable degeneration of additional cell populations within the basal ganglia, involving other neurotransmitter systems. ${ }^{8}$ Dyskinesiae developed in half of the treated patients. By contrast with patients with Parkinson's disease there was strikingly predominant involvement of orofacial and often neck muscles, with sustained dystonic elements in almost $60 \%$ of dyskinetic patients, which may reflect striatal somatotopy. Dyskinesiae also occurred in the absence of obvious improvement in motor function in some cases. ${ }^{\text {? }}$

Among patients still carrying a diagnosis of Parkinson's disease at death, we know that $24 \%$ do not have Parkinson's disease, and that multiple system atrophy is the most frequent cause of this error. ${ }^{17} 18$ Examining this question from a different angle, 12 of our 35 cases of multiple system atrophy remained misdiagnosed as Parkinson's disease until death. These constituted $34 \%$ of the entire series, and $55 \%$ of the cases not seen in life by us. Even retrospectively it was impossible in 10 of these cases to make a diagnosis of multiple system atrophy on the basis of the information recorded in their notes. The other two patients had developed pyramidal signs before death but remained diagnosed as Parkinson's disease. Although the presence of abnormal signs may suggest a diagnosis of multiple system atrophy, in many cases it may be the suspicion of multiple system atrophy that leads to the discovery of abnormal signs. Therefore a higher index of suspicion and regular follow up neurological examinations in all patients with parkinsonism may lead to more accurate diagnosis. Most cases of multiple system atrophy seem to start younger than is usual for Parkinson's disease, and it is of interest that the mean age of onset in the persistently misdiagnosed subgroup was significantly older than in the correctly diagnosed group. Even among those patients in whom an initial diagnosis of Parkinson's disease was subsequently changed to one of multiple system atrophy, this took an average of 4.4 years, indicating the difficulty of accurately diagnosing multiple system atrophy within the first five years. ${ }^{17}$

Our pathological study showed that both substantia nigra and putamen were severely depleted in most cases. In a few cases severe nigral atrophy was associated with mild or moderate putaminal degeneration and in two cases definite cell loss was restricted to substantia nigra and locus ceruleus only. ${ }^{12}$ The severity of pathological striatonigral degeneration did not correlate with age of onset and duration of disease, but did correlate with severity of disease at death.

Although $88 \%$ of our cases with available postmortem material had some evidence of olivopontocerebellar atrophy in addition to nigrostriatal cell loss, only $34 \%$ of them had definite cerebellar signs in life. Definite gait ataxia was only recorded in three of 12 cases with severe cerebellar (vermis) involvement, but impairment of postural reflexes with or without falls was present in all. The appreciation of a possible cerebellar component contributing to the disturbance of gait and 
postural control in multiple system atrophy is hampered by the presence of severe parkinsonism or symptomatic postural hypotension in many patients. The low number of cases with definite gait ataxia is therefore almost certainly an underestimate.

The association of olivopontocerebellar atrophy with striatonigral degeneration in such a large proportion of cases confirms the need for the term multiple system atrophy. ${ }^{1}$ In our series, all cases with adequately fixed brains contained oligodendroglial inclusions that have been reported by a number of groups in the brains of patients with multiple system atrophy of various clinical subtypes, and are rarely if ever found in other sporadic or hereditary neurodegenerative diseases including hereditary olivopontocerebellar atrophy. As in previous reports, in our series these inclusions were found not only in the olivopontocerebellar and striatonigral systems, but also in a number of areas not previously considered to be affected in this disease including the cerebral cortex, external capsule, and brainstem reticular formation..$^{919-22}$ The presence of these inclusions may account for more widespread alterations in cerebral function, such as the frontal lobe deficits documented in patients with multiple system atrophy. ${ }^{23-25}$ Furthermore, they constitute cytological grounds for considering sporadic striatonigral degeneration, Shy-Drager syndrome, and olivopontocerebellar atrophy together as multiple system atrophy, for differentiating them from hereditary olivopontocerebellar atrophy, and for defining multiple system atrophy as a specific entity that is only one among many different multisystem degenerations. ${ }^{26}$

GKW and SED are supported by the UK Parkinson's Disease Society, YBS by the Wellcome Trust, and MM by the Portuguese Health Service.

1 Graham JG, Oppenheimer DR. Orthostatic hypotension and nicotine sensitivity in a case of multiple system atrophy. F Neurol Neurosurg Psychiatry 1969;32:28-34.

2 Bannister R, Oppenheimer DR. Degenerative diseases of the nervous system associated with autonomic failure. Brain 1972;95:457-74.

3 Quinn N. Multiple system atrophy: the nature of the beast. f Neurol Neurosurg Psychiatry 1989;52(specialsuppl): 78 $\mathrm{Ne}$.

4 Oppenheimer DR. Diseases of the basal ganglia, cerebel- lum and motor neurons. In: Hume Adams J, Corsellis JAN, Duchen LW, eds. Greenfield's neuropathology. 4th ed. London: Arnold, 1984:699-747.

5 Daniel SE. The neuropathology and neurochemistry of multiple system atrophy. In: Bannister R, Mathias CJ, eds. Autonomic failure. A textbook of clinical disorders of the autonomic nervous system. 3rd ed. Oxford: Oxford University Press, 1992:564-85.

6 Fearnley JM, Lees A. Striatonigral degeneration. A clinicopathological study. Brain 1990;113:1823-43.

7 Hughes AJ, Colosimo C, Kleedorfer B, Daniel SE, Lees AJ. The dopaminergic response in multiple system atroAJ. The dopaminergic response in multiple system atro-

8 Quinn N. Multiple system atrophy. In: Marsden CD, Fahn S, eds. Movement disorders 3. London: Butterworths-Heinemann Ltd, 1994:262-81.

9 Papp MI, Kahn JE, Lantos PL. Glial cytoplasmic inclusions in the CNS of patients with multiple system atrophy (striatonigral degeneration, olivopontocerebellar atrophy and Shy Drager syndrome). $\mathcal{F}$ Neurol Sci 1989;94:79-100.

10 Lenter C. Geigy scientific tables Vol 2. Basle: Ciba-Geigy, 1982.

11 Hoehn MM. Parkinsonism treated with levodopa: progression and mortality. $\mathcal{f}$ Neural Transm 1985; 19(suppl):253-64

12 Wenning GK, Quinn N, Magalhães M, Mathias C, Lees Wenning GK, Quinn N, Magalhães $M$, Mathias $C$, Lees phy. Mov Disord 1994;9:161-6.

13 Adams RD, van Bogaert $L$, van der Eecken $H$. Dégénérescences nigro-striées et cérébello-nigro-striées. Psychiatria et Neurologia 1961;142:219-59.

14 Dejerine J, Thomas AA. L'atrophie olivo-ponto-cérébelleuse. Nouvelle Iconographie de le Salpêtrière 1900;13: 330-70.

15 Shy GM, Drager GA. A neurologic syndrome associated with orthostatic hypotension. Arch Neurol 1960;2: 511-27.

16 Wenning GK, Ben Shlomo Y, Magalhães M, Daniel SE, Quinn NP. Clinical features and natural history of multiple system atrophy: an analysis of 100 cases. Brain 1995;117:835-45.

17 Rajput AH, Rozdilsky B, Rajput A. Accuracy of clinical diagnosis in parkinsonism-a prospective study. Can $\mathcal{F}$ diagnosis in parkinsonism
Neurol Sci 1991;18:275-8.

18 Hughes AJ, Daniel SE, Kilford L, Lees A. The accuracy of clinical diagnosis of idiopathic Parkinson's disease: a clinicopathological study. $\mathcal{F}$ Neurol Neurosurg Psychiatry 1992;55:181-4

19 Nakazato Y, Yamazaki H, Hirato J, et al. Oligodendroglial microtubular tangles in olivopontocerebellar atrophy. $\mathcal{f}$ Neuropathol Exp Neurol 1990;49:521-30.

20 Costa C, Duyckaerts C, Cervera P, et al. Les inclusions oligodendriales, un marqueur des atrophies multisystématisées. Rev Neurol 1992;148:274-80.

21 Mochizuki A, Mizusawa H, Ohkoshi N, et al. Argentophilic intracytoplasmic inclusions in multiple system atrophy. $¥$ Neurol 1992;239:311-6.

22 Papp MI, Lantos PL. Accumulation of tubular structures in oligodendroglial and neuronal cells as the basic alteration in multiple system atrophy. $\mathcal{F}$ Neurol Sci 1992; ation in multip

23 De Volder AG, Francart J, Laterre C, et al. Decreased glucose utilization in the striatum and frontal lobe in probable striatonigral degeneration. Ann Neurol 1989, 26:239-47.

24 Eidelberg D, Takikawa S, Moeller JR, et al. Striatal hypometabolism distinguishes striatonigral degeneration from Parkinson's disease. Ann Neurol 1993;33:518-27.

25 Robbins TW, James M, Lange KW, Owen AM, Quin NP, Marsden CD. Cognitive performance in multiple system atrophy. Brain 1992;115:271-91.

26 Lantos PL, Papp MI. Cellular pathology of multiple system atrophy. F Neurol Neurosurg Psychiatry 1994;57: 129-33. 